\title{
Video over DSL with LDGM Codes for Interactive Applications
}

\author{
Filippo Casu and Julián Cabrera
}

\author{
Laith Al-Jobouri and Martin Fleury
}

\begin{abstract}
Digital Subscriber Line (DSL) network access is subject to error bursts, which, for interactive video, can introduce unacceptable latencies if video packets need to be resent. If the video packets are protected against errors with Forward Error Correction (FEC) calculation of the applicationlayer channel codes themselves may also introduce additional latency. This paper proposes Low-Density Generator Codes (LDGM) rather than other popular codes because they are more suitable for interactive video streaming not only for their computational simplicity but also for their licensing advantage. The paper demonstrates that up to a $4 \mathrm{~dB}$ reduction in video distortion is achievable with LDGM Application Layer (AL) FEC. Telemedicine and video conferencing are typical target applications.
\end{abstract}

Keywords-DSL; FEC; low latency; packet loss; video streaming

\section{INTRODUCTION}

Compressed video has recently received a boost from around a $50 \%$ reduction in bandwidth requirements arising from the High Efficiency Video Coding (HEVC) codec standard [1]. Unfortunately, either owing to a desire to reduce codec complexity or owing to a preference for commercial high-definition video-on-demand applications, low-latency video streaming is no longer strongly supported in the codec. As a result, applications such as telemedicine and video conferencing have a limited range of built-in error-resilience tools available [2]. Further, the Dynamic Adaptive Streaming over HTTP (DASH), which is supported by HEVC, owing to the underlying Transmission Control Protocol (TCP) used by DASH introduces packet re-sending delay whenever a packet is lost to errors or congestion. Video streaming has an important role in telemedicine [3] both in longer-term monitoring and in emergency responses, where the need for low-latency communication is probably strongest. However, for home advice it would be helpful if face-to-face consultation with a remote clinician were possible. This assumes that HEVC encoding delays, which are currently many times above realtime [4], can be addressed.

For interactive video streaming, with an associated speech channel, delay is harmful to synchronization at both ends of the communication link. Digital Subscriber Line (DSL) is the dominant broadband access network for residential users with 364.1 million links in 2012 [5]. However, impulse noise is a potent source of DSL transmission errors, resulting in fixedlength error bursts, resulting in multiple packet losses, depending on transmission rate. Sources of error bursts vary from street lights though faulty set-to boxes and even from flashing Christmas tree lights [6]. As a way of responding to packet error bursts, end-to-end Automatic Repeat ReQuest (ARQ) packets introduce latency, particularly if used from endto-end, leaving Forward Error Correction (FEC) as the main way of reducing errors in the absence of effective error resilience tools.

This paper considers an application-layer (AL) FEC solution to the problem of video packet loss on DSLs. If packets are lost, due to video coding dependencies, video streams may be disrupted for up to $30 \mathrm{~s}$, the duration of a typical Group of Pictures (GoP) [7]. Consequently, PHYsicallayer FEC protection in the case of video is often supplemented by AL-FEC [8], which has been introduced into the main wireless standards. Typical examples discussed in Section II of FEC codes [9] employed in packetized video streams are: eXclusive OR (XOR)-based codes, simple or interleaved; LowDensity Parity-Check (LDPC) codes; and Reed-Solomon (RS) codes.

However, FEC or channel codes due to their computational complexity may also introduce latency. Coding latency can be composed of: the need to accumulate sufficient data to successfully repair packets, a problem that may arise with the rateless codes [10], including Raptor codes [8], leading to large input buffers; and the delay arising from the computational complexity of the coding and decoding operations, a problem with Reed-Solomon (RS) codes [11] as the block size increases. When rateless codes are employed adaptively another source of latency may arise, owing to a need to request the sender to stop sending additional repair packets. Though there are now open source versions of Raptor code, Raptor 10 and RaptorQ [12], for research purposes, care should be taken not to infringe any of the patents associated with Raptor coding. Thus, to computational complexity, in some cases, one can add legal complexity.

Interleaving of packets in order to reduce the impact of error bursts also has a latency implication, again owing to the 
need to accumulate sufficient data before interleaving can take place. In addition, whenever the latency budget is large, the video sending rate becomes 'bursty', which can lead to congestion and ultimately to packets being dropped from buffers. Therefore, some of the results presented in this paper, examine the trade-off between error repair capability and the latency arising from a choice of FEC method. As it is, because the packet loss pattern, rather than the number of packets lost, actually affects video distortion, other results examine the video delivered after using our recommended low-latency channel coder.

To reduce coding latency, Low-Density Generator Matrix (LDGM) codes [13], a lower-complexity version of LDPC codes, are attractive candidates. They are block-based but unlike LDPC codes, which can have block sizes of about 1000 for best recovery performance. Similarly, their reduced coding complexity compared to LDPC codes can be traded against their error-correction performance. Unlike RS codes, decoding can be iteratively refined through a belief-propagation algorithm, rather than having to wait for all the data before decoding can begin. As in both [14] and [15], LDPC was selected as most suitable for Real-time Transport Protocol (RTP) video streaming compared to other channel codes, the possibilities for its simplified cousin, LDGM, are promising. In this paper, we now consider the relevance of LDGM codes for video communication with shorter block lengths, whereas work in [13] considered their relevance for general data communication with large block lengths.

The remainder of this paper is organized as follows. Section II reviews the advantages and disadvantages of various channels codes suitable for protection of real-time video streams. Section III is a comparison across those codes in terms of the trade-offs to be made across the dimensions of latency, error recovery, severity of error conditions, and resulting video distortion. Finally, Section IV summarizes the paper.

\section{ChanNel CODES}

This Section is a review of available channel codes from the points of view of latency, computational complexity, error correction capability, and other implementation factors.

\section{A. $L D P C$ codes}

LDPC codes are linear block codes [16] characterized by parameters $k$, and $n$, which correspond to the number of bits (assuming for the moment a bit-sized symbol) of an information and code vector respectively. Therefore, the number of redundant bits is $n-k$. An LDPC code is defined by its parity matrix $H$ of dimensions $n-k$ by $n$, such that:

$$
\mathbf{c} H^{T}=\mathbf{0}
$$

where $\mathbf{c}$ is a code vector. As the code name implies, matrix $H$ has a low density of 1's. LDPC (and its lower-complexity cousin LDGM) commonly employs a regular, staircase matrix layout [13], in which the number of 1's in each row and column is constant. $H$ provides $n-k$ parity check equations that generate constraints between data symbols and parity symbols. These constraints indicate which data symbols are involved in the XOR operations to generate the parity symbols. From $H$, the generator matrix $G$ can be constructed to generate redundant data from the information vector $\mathbf{u}$ :

$$
\mathbf{c}=\mathbf{u} G
$$

Although LDPC codes are not a Maximum-Distance Separable (MDS), implying that they do not offer the optimal recovery capability for a block code, they already have a low computational complexity. That characteristic occurs for two reasons: 1) the use of XOR operation to generate the redundancy; and 2) the low density of the generator matrix, which results in a low number of operations.

\section{B. LDGM codes}

A simplified version of LDPC codes is represented by LDGM codes, for which the parity-check matrix $H$ corresponds to the generator matrix $G$ [13]. LDGM codes have several potential advantages despite the reduction in recovery performance: as the complexity is even lower than LDPC codes, they are suited to encoding/decoding on a variety of battery-powered mobile devices; and low values of $k$ imply that for real-time video applications such as telemedicine or video conferencing, the latency budget is considerably reduced. In the LDGM approach, the parity-check matrix $H$ has a size of $n-k$ by $k$, compared to the LDPC case in Section II.A of $n-k$ by $n$. Therefore, only the first $k$ symbols and their coding contribution are taken into consideration, compared to the LDPC case, for which $n$ symbols have to be processed. Finally, they appear to have very low legal complexity.

There is a downside: LDGM codes have high error floors that cannot necessarily be reduced by increasing the block size. (When, after application of FEC, the bit error rate ceases to reduce with decreased Signal-to-Noise Ratio (SNR), an error floor is said to exist.) However, at least for a binary symmetric channel (BSC) it is has been analytically demonstrated [17] that concatenating two LDGM codes (applying one LDGM code after another) overcomes the onset of an error floor, while retaining LDGM's computational complexity, provided a belief-propagation (message-passing) decoding algorithm is employed. Later work [18] confirmed the findings of [17] for a Rayleigh channel and provides analysis on how best to configure LDGM codes. In this paper, we simulate an erasure channel, which is not necessarily open to analysis in the way a BSC is but nevertheless occurs in practice after PHY-layer error recovery fails to recover a packet. An erasure channel is distinguished by the property that the positions of corrupted symbols are known in advance, usually because an upper-layer protocol records the packet sequence number.

\section{Pro-MPEG COP\#3}

Professional-MPEG code of practice \#3 (Pro-MPEG COP \#3) [19] is an industry standard for video transmission protection that is widely deployed. Incoming packets are 
arranged in a matrix on a row-by-row basis, assuming packetsized symbols. Redundant packets are subsequently appended to each column of the matrix and optionally to each row of packets. The packets are transmitted column-by-column, i.e. orthogonally to the way they were read into the matrix. The redundant packets are created by a byte-wise XOR operation across the packets of each column/row. This simple interleaving scheme has the advantage of convenient hardware implementations. The standard restricts the number of columns and rows to a maximum of 20 .

In this paper, the one-dimensional version of Pro-MPEG COP \#3 is tested in which redundant packets are only created for the columns. One-dimensional Pro-MPEG COP \#3 was also selected in [10] for the reason that it is more widely deployed. The number of rows was set to four (the minimum) and the number of packets in a column to 20. This is the same configuration as employed in [20] as part of an unequal loss protection (ULP) scheme, according to the video frame type (IB- and P-type) priority. In the current paper, for ease of comparison, ULP is not used.

\section{D. $R S$ codes}

RS codes have the MDS property, i.e. any $k$ packets can be received to recover the $k$ information packets, whereas around $\mathrm{k} \times 1.05 \%$ of packets are needed [15] for full recovery in LDPC. RS codes are linear, cyclic codes, formed by sequences of $m$-bits symbols, each of which symbols belong to a finite Galois Field, i.e. GF $\left(2^{m}\right)$, where $m$ takes values greater than two. $n$ is set to the value $2^{m}-1$. If $m$ is greater than eight the RS computational complexity can be prohibitive. Specifically, the total complexity is $\mathrm{O}\left((k /(n-k)) \times(\log (k))^{2}+\log (k)\right)$, even when using a fast frequency-domain algorithm [21].

RS FEC in the common intra-packet approach works by grouping $k$ packets at a time. From each of the $k$ packets, the first $\mathrm{m}$-bits are extracted to form $k \mathrm{~m}$-bit symbols. These $k$ symbols are employed to generate $n-k$ redundant symbols by means of the RS algorithm. The redundant symbols are then packed, as the first $m$-bit symbols of $n-k$ parity packets. The intra-packet algorithm continues by extracting the next set of $m$-bit symbols and forming $n-k m$-bit redundant symbols and packing as the next set of symbols in the $n-k$ parity packets.

In [22], the interleaving factor was increased by forming each $m$-bit symbol by extracting one bit from each of $m$ packets in turn. This alternative inter-packet approach improves the error recovery performance in bursty error conditions, as the loss of any one symbol affects only one bit per packet. However, whereas the latency budget of the intra-packet scheme is the time for $k$ packets to arrive, in the inter-packet budget it is the time for $k \times m$ packets to arrive at the sender.

\section{FINDINGS}

This Section firstly compares across the candidate channel codes before investigating how the proposed LDGM method performs when applied specifically to video streams. In the experiments, the code was a regular LDGM code with degree three (see Section II.A on such matrices mentioned in relation to LDPC). The generator matrix was created by using the classical pseudo-random algorithm. The decoding was based on the belief-propagation iterative algorithm mentioned in Section I.

\section{A. Comparison across channel codes}

Packet error bursts were simulated by a Gilbert-Elliott twostate, hidden Markov model [23], in which a worst-case Packet Error Rate (PER) for ADSL was taken to be 1\% [8], i.e. the bad state of the Gilbert-Elliott model was entered on average about $1 \%$ of time. Bursts occurred randomly (Uniform distribution). Burst lengths in time are around $8 \mathrm{~ms}$ [8] according to the Repetitive Electrical Impulse Noise (REIN) model for ADSL channels. The packet error burst lengths (Ln), which depend on the transmission rate, were set to 15,20 , and 25 . The simulation ranged over the video data from a two-hour video movie.

An ADSL packet size was set to 50 bytes (B). That size is close to the $53 \mathrm{~B}$ cell size of the Asynchronous Transfer Mode (ATM) [24] predominantly employed at the data-link layer over ADSL. (Only 48 bytes (B) form the payload in ATM; the remaining header bytes are heavily protected.). ADSL "Fast Track" [25] was turned on but packet interleaving to reduce further the impact of error bursts was turned off so as not to introduce additional latency. ADSL transmission rates vary according to the version of ADSL. For downstream transmission a maximum of $8 \mathrm{Mbps}$ is achievable in the earliest version, rising to a projected $52 \mathrm{Mbps}$ in the recent ADSL2++. However, it is the upstream transmission rate that is limiting for interactive video streaming and this correspondingly ranges from a maximum of $1 \mathrm{Mbps}$ to a projected $5 \mathrm{Mbps}$.

In this evaluation, as previously mentioned, an erasure channel was assumed. In practice, groups of erasures would need to be detected at the application-layer if that layer was used to identify which packets forming a video bitstream had been lost. MPEG2-Transport Stream (TS) [26] (a standard way of encapsulating multimedia data) has a packet size is $\mathbf{1 8 8}$ bytes, of which four bytes form a header. The MPEG2-TS headers contain a 13-bit packet identifier, which can be employed to identify erasures. Multiple MPEG2-TS packets can be packed into RTP packets for core network transport. RTP packets also contain a sequence number in bits $16-31$ of the header but up to eight MPEG-2 packets are typically contained in an RTP packet, limiting its header's use in detecting erasures.

The coding rate, $k / n$, was set to $90 \%$ with $n=550$. In order to make the RS intra-packet coding rate the same for ProMPEG COP \#3 and LDGM codes, the RS parameter $m$ was set to 6 , not 8 as is more usual. For RS coding, the packet stream was grouped 80 packets at a time. The Pro-MPEG COP \#3 configuration was described in Section II.

From Figure 1, it is apparent that inter-packet RS [22] has the highest error-recovery property, which will also be higher than the standard intra-packet method. However, the industry standard Pro-MPEG COP \#3 deteriorates sharply in its error recovery capability when the burst length increases. At packet burst lengths of 20 and 25 packets, LDGM provides better error recovery properties than Pro-MPEG COP \#3. Moreover, the Pro-MPEG COP \#3 latency budget is much longer in duration of time than LDGM because all packets in Po-MPEG 


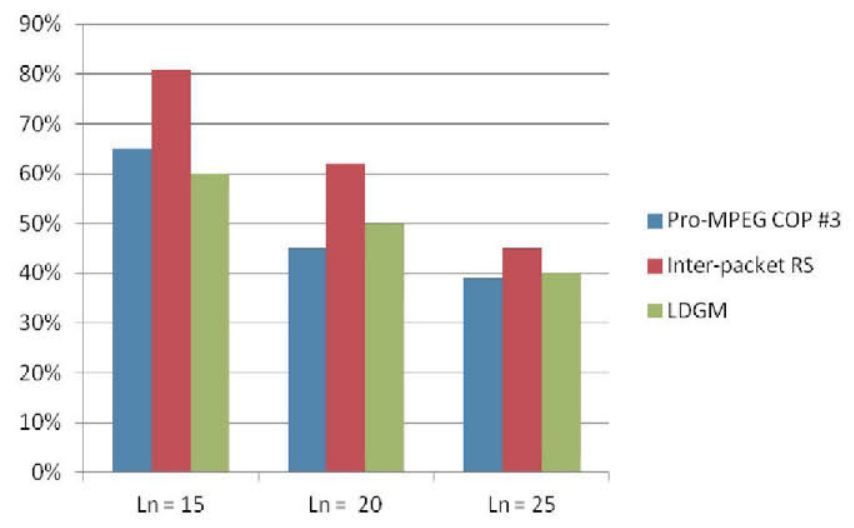

Figure 1. Percentage of recovered packets with PER $=1 \%$ and increasing packet burst lengths ( $\mathrm{Ln}$ ) for three channel codes.

COP \#3's interleaving matrix must first be assembled at the encoder (and likewise arrive at the decoder). Similarly, for RS coding there is a latency budget of k packets at the encoder for the intra-packet version and k.m for the evaluated inter-packet version. Moreover, as previously mentioned, when using LDGM, decoding can begin at an early stage when applying the belief-propagation algorithm. The computational complexity of the RS algorithm, according to Section II, is much higher than that of LDGM.

\section{B. Impact upon video distortion from $L D G M$}

In experiments in this Section, ADSL was again assumed with small packet sizes of $50 \mathrm{~B}$ and $100 \mathrm{~B}$ for each of two sets of tests respectively. The ADSL configuration was the same as in the Section III.B. However, the results from testing with a larger $100 \mathrm{~B}$ packet size are also included in this Section, bringing the packet size closer to that of an MPEG2-TS packet. For $100 \mathrm{~B}$ packets a downstream bitrate of $10 \mathrm{Mbps}$ was configured, with a per-packet link latency of around $100 \mathrm{~ms}$. For $50 \mathrm{~B}$ packets and two-way communication a $1 \mathrm{Mbps}$ effective datarate was assumed, with a per-packet link latency of around $10 \mathrm{~ms}$. As previously, the PER was set $1 \%$ [8], though with burst lengths of 8 and 10 packets for the $50 \mathrm{~B}$ and $100 \mathrm{~B}$ packets respectively. Thus, the channel conditions were more benign than in the previous Section's experiment.

To counter error bursts, the packet block size was set to $k=$ 300,400 . $n-k$, the number of redundant packets, was somewhat reduced to $9 \%$ of the whole. The latency budget remains well below the previously mentioned 1000 packets length of large block coding schemes.

To provide a realistic evaluation of video distortion, tests used the reference video sequence Football, with plenty of motion activity, which increases the temporal compression coding dependencies. In order to judge the video distortion, a video trace was fed into a numerical simulator (refer to Figure 2) where ADSL packetization took place. After numerical simulation, data from the ADSL packets judged lost were removed from the compressed video bitstream, prior to passing through the H.264/Advanced Video Coding (AVC) decoder. The resulting bitstreams (before and after LDGM repair) were compared to the YUV video input to determine the Peak Signal-to-Noise Ratio (PSNR).

To allow the gain from combining FEC with built-in error resiliency, HEVC was not used. Instead the video sequence was encoded with the H.264/AVC [27] JM 14.2 codec in Common Intermediate Format $(352 \times 288$ pixels/frame $)$ at 30 frames/s at a constant bitrate (CBR) of $1 \mathrm{Mbps}$. The frame structure was an initial intra-coded frame followed by all predictively-coded P-frames. $2 \%$ intra-coded macroblocks (MBs) were included in the P-frames to guard against temporal error propagation. The IPPP... frame structure with intra-coded MBs included, is also suitable for streaming to mobile devices, as there is reduced computation because bi-predictive B-frames are no longer employed. Channel switching, for which periodic I-frames are useful, is not expected in a telemedicine or videoconferencing application. Data partitioning was also turned on at the codec as an additional form of error resilience, with constrained intra prediction also configured. These video settings conform to the recommendations of [28].

Table I shows 5 sample runs each with a different seed and the resulting mean PSNR. (The code seed was set to 40, 50, 70, 80 , and 90 for $1-5$ respectively.) The mean gain after application of LDGM was between 1 and $2 \mathrm{~dB}$ with the video

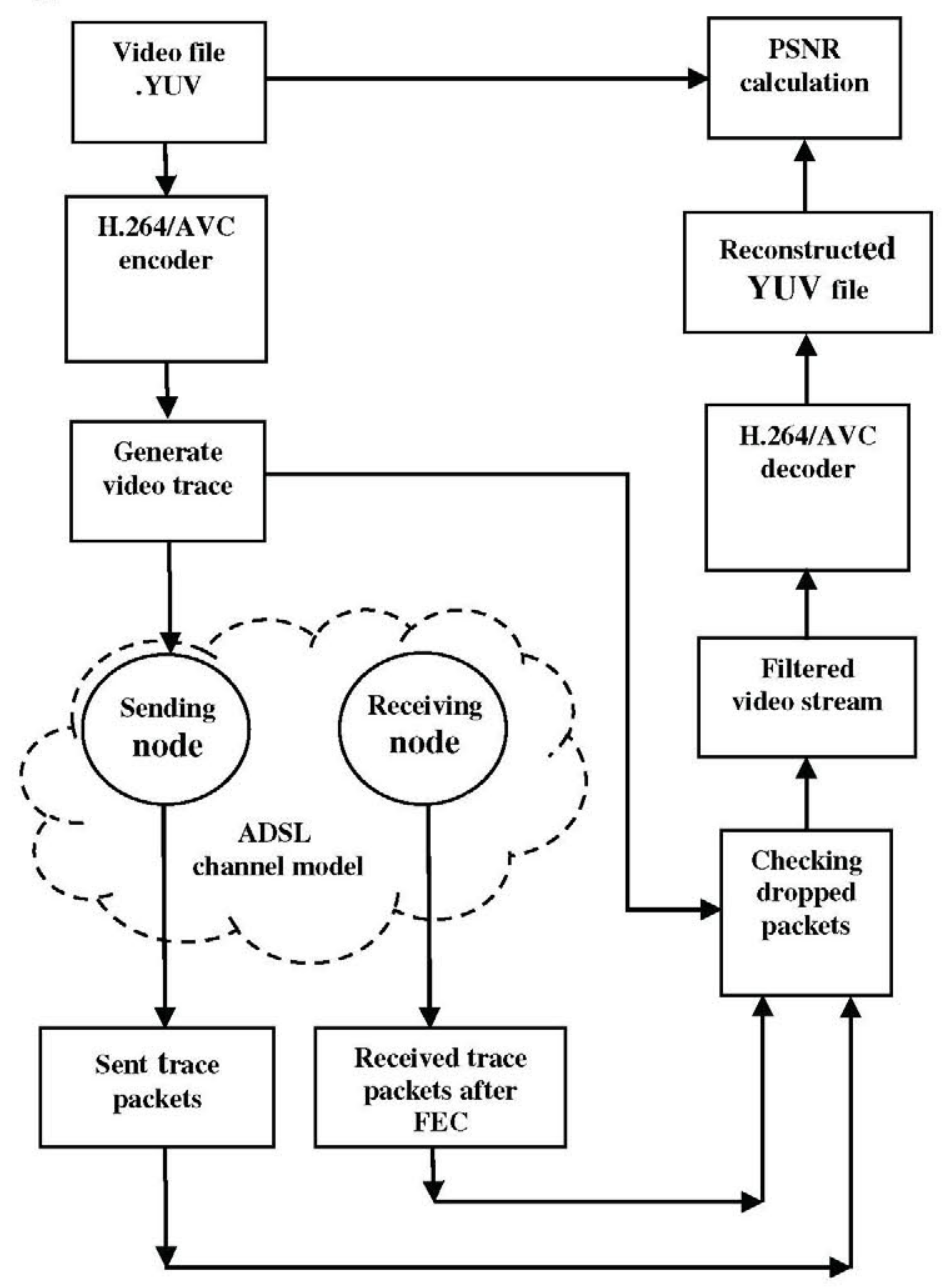

Figure 2. PSNR evaluation framework. 
TABLE I

OBJECTIVE VIDEO PSNR FOR 50 B PACKETS BEFORE AND AFTER FEC

\begin{tabular}{|c|c|c|c|c|c|c|}
\hline $\begin{array}{c}\text { Before } \\
\text { FEC }\end{array}$ & 1 & 2 & 3 & 4 & 5 & $\begin{array}{c}\text { Mean PSNR } \\
(\mathrm{dB})\end{array}$ \\
\hline $\mathrm{k}=300$ & 35.53 & 35.08 & 38.63 & 36.14 & 37.37 & 36.55 \\
\hline $\mathrm{k}=400$ & 34.46 & 37.66 & 38.54 & 36.40 & 39.00 & 37.21 \\
\hline After & 1 & 2 & 3 & 4 & 5 & \\
\hline $\mathrm{k}=300$ & 37.33 & 39.00 & 39.00 & 37.57 & 37.84 & 38.15 \\
\hline $\mathrm{k}=400$ & 36.91 & 37.76 & 39.00 & 37.64 & 39.00 & 38.06 \\
\hline
\end{tabular}

TABLE II

OBJECTIVE VIDEO PSNR FOR 100 B PACKETS BEFORE AND AFTER FEC

\begin{tabular}{|c|c|c|c|c|c|c|}
\hline $\begin{array}{c}\text { Before } \\
\text { FEC }\end{array}$ & 1 & 2 & 3 & 4 & 5 & $\begin{array}{c}\text { Mean PSNR } \\
(\mathrm{dB})\end{array}$ \\
\hline $\mathrm{k}=200$ & 34.53 & 33.95 & 34.91 & 34.93 & 35.64 & 34.79 \\
\hline $\mathrm{k}=300$ & 36.54 & 36.41 & 33.81 & 34.69 & 32.84 & 34.86 \\
\hline After & 1 & 2 & 3 & 4 & 5 & \\
\hline $\mathrm{k}=200$ & 38.22 & 36.67 & 39.00 & 39.00 & 38.86 & 38.35 \\
\hline $\mathrm{k}=300$ & 38.85 & 36.55 & 39.00 & 38.88 & 37.35 & 38.13 \\
\hline
\end{tabular}

PSNR approaching a level suitable for broadcast. Interestingly from the point of view of latency, increasing the block size does not necessarily lead to a reduction in video distortion.

For the larger packet size and the greater bandwidth of Table II, the video distortion reduction is more consistent and is $3-4 \mathrm{~dB}$. The consistency is due to a constant code seed of 50 throughout. Notice that in view of the larger $100 \mathrm{~B}$ packet size the block sizes are decreased. Again a larger block size appears not to lead to an advantage. This effect may be linked to the pattern of packet burst erasures. Comparing the $100 \mathrm{~B}$ PSNR gain to that of $50 \mathrm{~B}$ packets, for the latter the FEC gain appears to have saturated, suggesting a reduced FEC rate is possible.

\section{RELATED WORK}

The impact of impulse noise on an ADSL2+ link is documented in [29]. Evidently there is severe 'blockiness', where runs of macroblocks (MBs) have been lost and error concealment at the codec decoder has failed to replace the MBs in an unobtrusive manner.

In [15], a simple 2D FEC code (with similarities to ProMPEG COP \#3), RS codes, and LDPC were evaluated by embedding them in Linux RTP protocol stacks that included RTP packetization with IP/UDP headers. Packet erasures were uniformly distributed. The coding rate was high at $2 / 3$. The authors reported that above $30 \%$ PERs, no codes could repair all packets but LDPC was only slightly worse than RS in error recovery. The simple $2 \mathrm{D} \mathrm{FEC} \mathrm{code} \mathrm{was} \mathrm{noticeably} \mathrm{worse} \mathrm{than}$ both LDPC and RS. Comparing latencies, RS codes introduced maximum average delay of $544 \mathrm{~ms}$, followed by LDPC with 1000 block lengths at $402 \mathrm{~ms}$ and the simple 2D FEC codes at around $50 \mathrm{~ms}$. Lower block lengths for LDPC reduced latency but decreased error recovery. These results confirm that a compromise code, such as LDGM (or low block length LDPC), are a good option for interactive video. Computational overhead for the simple 2D FEC code and $\mathrm{LDPC}$ remained below $8 \%$ whatever the PER while as the error rate increased RS code computational overhead climbed steeply.

As part of the OpenFEC project, [30] examined the same codes as in [15] but with measured error traces from ADSL links rather than the random drops of [15]. These results confirmed those of [15] for random losses but showed that even at loss rates below $10 \%$ and with a code rate of $2 / 3$, when error bursts occurred not all packets could be recovered. As in [15] an LDPC code with a block length as low as 170 was found to be competitive with RS codes in error recovery terms. Because not all packets could be recovered even at low loss rates, the ability to retransmit was recommended, which is also the recommendation of [28].

\section{CONCLUSION AND FUTURE WORK}

This study has shown study that industry-standard 2D parity codes underperform in terms of combined latency and error recovery. RS codes are attractive in terms of error recovery but are not so attractive for low-latency applications of video streaming. Whenever there is interactivity RS codes at the application layer potentially result in a lack of synchronization between two communicating parties. This problem arises owing to their computational complexity and the need to apply them in an interleaving mode, which increases the latency budget. As an alternative, this paper proposes that LDGM codes with small block lengths represent a natural candidate for low-latency interactive video streaming and, as results quoted in this paper indicate, can lead to up to a $4 \mathrm{~dB}$ reduction in video distortion for active sports sequences. The coding overhead is just a $9 \%$ increase in datarate. Given that rateless codes may have patents applied, LDGM codes offer a further commercial advantage, because licensing fees no longer apply. The latter advantage makes LDGM codes suitable for applications such as telemedicine in which video streaming does not generate a compensating revenue stream.

Future work will check the performance of these codes against video content other than the sports sequences so far investigated. The HEVC codec is aimed at high definition (HD) video and, hence, the HD video should also be investigated, confirming the real-time performance. In general, as the number of packets in $\mathrm{HD}$ video communication is much larger than for standard definition (SD) video the impact of any one packet loss in terms of error propagation is expected [31] to be less than that for SD video. Consequently, a pure FEC technique such as in this paper may be even more effective for $\mathrm{HD}$ video. In general, the real-time transmission and FEC coding time will scale linearly according to the number of packets in an HD video frame relative to an SD frame. Thus in [31] there were 31 and 68 rows of macroblocks for SD and HD respectively, with coding at rates of 4 and $16 \mathrm{Mbps}$ respectively using H.264/AVC. HEVC is expected [1] to be approximately $50 \%$ more efficient at coding than H.264/AVC but paradoxically, while saving bandwidth could reduce the impact of LDGM FEC. 


\section{REFERENCES}

[1] J. Ohm, G. Sullivan, H. Schwarz, and T.K. Tan, "Comparison of the coding efficiency of video coding standards - Including High Efficiency Video Coding (HEVC)," IEEE Trans. Circuits Syst. Video Technol., vol. 22, no. 12, pp. 1669-1684, 2012.

[2] T. Schierl,M.M. Hannuksela, Y.-W. Wang, and S. Wenger, "System layer integration of High Efficiency Video Coding," IEEE Trans. Circuits Syst. Video Technol., vol.22, no. 12, pp. 1871-1884, 2012.

[3] A. Panayides, M.S. Pattichis, C.S. Pattichis, C.P. Loizou, M. Pantziaris, and A. Pitsillides, "Robust and efficient ultrasound video coding in noisy channels using H.264," in IEEE Ann. Int. Conf. of Eng. in Medicine and Biology Society, 2009, pp. 5143-5146.

[4] F. Bossen, B. Bross, K. Sühring, and D. Flynn, "HEVC complexity and implementation analysis," IEEE Trans. Circuits Syst. Video Technol., vol. 22, no. 12, pp. 1685-1696, 2012.

[5] Point Topic Ltd., "World Broadband Statistics: Q2, 2012" London, U.K., 2012.

[6] British Telecom (BT) Wholesale, "REIN - Repetitive Electrical Impulse Noise Customer Introduction," version 1.0, 2013.

[7] M. Ghanbari, Standard codecs: Image compression to advanced video coding, Stevenage, UK: Institute of Electrical Engineers (now IET), 2003.

[8] M. Luby, T. Stockhammer, and M. Watson, "Application layer FEC in IPTV services," IEEE Commun. Mag., vol. 46, no. 5, pp. 94-101, 2008.

[9] C. Lamoriniere, A. Nafaa, and L. Murphy, "Dynamic switching between adaptive FEC protocols for reliable multi-source streaming," in IEEE GLOBECOM, 2009

[10] A.C. Begen, "Error Control for IPTV over xDSL networks," in IEEE Int. Conf. on Consumer Commun. and Networking Conf., 2008, pp. 632637.

[11] T. Jokela, and E. Lehtonen, "Reed-Solomon decoding algorithms and their complexities at the DVB-H link-layer," in $4^{\text {th }}$ IEEE Int. Symp. Wireless Commun. Systems, 2007, pp. 752-756.

[12] A. Shokrollahi, and M. Luby, "Raptor codes," Foundations and Trends in Commun. and Info. Theory, vol. 6, nos. 3-4, pp. 213-322, 2009.

[13] V. Roca, Z. Khalluf, and J. Labouré, "Design and evaluation of an LDGM large block FEC codec," in Fifth Int. Workshop on Networked Group Commun., 2003.

[14] V. Pullano, R. Skupin, G.E. Corazza, C. Hellge, and T. Schierl, "Modeling effects of impulse noise on application-layer FEC in DSL channels," in IEEE Int. Symp. Broadband Mult. Syst. Broadcast., 2013.
[15] K. Matsuzono, J. Detchart, M. Cunche, V. Roca et al. "Performance analysis of a high-performance real-time application with several ALFEC schemes," in IEEE Local Computer Networks, 2010.

[16] W. E. Ryan, "An introduction to LDPC Codes," in CRC Handbook for Coding and Signal Processing for Recording Systems, B. Vasic and E. Kurtas, Eds., Boca Raton, FL: CRC Press, 2004.

[17] J. Garcia-Frias, and W. Zhong, "Approaching Shannon performance by iterative decoding of linear codes with Low-Density Generator Matrix," IEEE Commun. Lett., vol. 7, no. 6, pp. 266-269, 2003.

[18] M. González-López, F. J. Vázquez-Araújo, L. Castedo, and J. GarciaFrias, "Serially-concatenated Low-Density Generator Matrix (SCLDGM) codes for transmission over AWGN and Rayleigh fading channels," IEEE Trans. on Wireless Commun., vol. 6, no. 8, pp. 27532758,2007

[19] Pro-MPEG Forum, "Transmission of Professional MPEG-2 transport streams over IP networks," Code of Practice \#3 release 2, July 2004.

[20] C. Díaz, J. Cabrera, F. Jaureguizar, and N. García, "A video-aware FECbased unequal loss protection system for video streaming over RTP," IEEE Trans. Consum. Electron., vol. 57, no. 2, pp. 523-531, 2011.

[21] J. Lacan, V. Roca, J. Peltotalo, and S. Peltotalo, "Reed-Solomon Forward Error Correction (FEC) schemes," IETF RFC 5510, 2009.

[22] F. Casu, J. Cabrera, F. Jaureguizar, and N. García, "Inter-packet symbol approach to Reed-Solomon FEC codes for RTP-multimedia stream protection," in IEEE Symp. on Comput. and Commun., 2011, pp. 49-54.

[23] C. Jiao, L. Schwiebert, and B. Xu, "On modeling the packet error statistics in bursty channels," in IEEE Conf. on Local Computer Networks, 2002, pp. 534-541.

[24] M. De Prycker, Asynchronous Transfer Mode. Solutions for Broadband ISDN, Upper Saddle River, NJ: Prentice Hall, 1993.

[25] W.J. Goralski, ADSL and DSL Technologies (Standards \& Protocols) $\left(2^{\text {nd }}\right.$ ed.), Boston, MA: McGraw-Hill, 2001.

[26] J.-N. Hwang, Multimedia networking: From theory to practice, Cambridge, UK: Cambridge University Press, 2009.

[27] S. Wenger, "H.264/AVC over IP," IEEE Trans. Circuits Syst. Video Technol., vol. 13, no. 7, pp. 645-655, 2003.

[28] L. Al-Jobouri, M. Fleury, and M. Ghanbari, "Engineering wireless broadband access to IPTV," $J$. of Visual Commun. and Image Representation, vol. 25, no. 7, pp. 1493-1506, 2014.

[29] J. Krejčí, and T. Zeman, "Influence of noise on IPTV over xDSL," in $16^{\text {th }}$ Int. Conf. on Systems, Signals and Image Process., 2009.

[30] M. Ellis, D.P. Pezaros, and C. Perkins, "Performance analysis of ALFEC for RTP-based streaming video traffic to residential users," in IEEE 19th Int. Packet Video Workshop, 2012.

[31] S. Argyopoulos, A. Raake, M.-N. Garcia, and P. List, "No reference bit stream model for video quality assessment of H.264/AVC video based on packet loss visibility," IEEE Int. Conf. Acoustics, Speech, Signal Process, 2011, pp. 1169-1172. 